\title{
Cell-based therapy in thin endometrium and Asherman syndrome
}

\author{
Nastaran Gharibeh ${ }^{1,2 \dagger}$, Leili Aghebati-Maleki ${ }^{3 \dagger}$, Javad Madani ${ }^{2}$, Ramin Pourakbari ${ }^{1,2}$, Mehdi Yousefi ${ }^{2,5^{*}}$ and \\ Javad Ahmadian Heris ${ }^{4 *}$
}

\begin{abstract}
Numerous treatment strategies have so far been proposed for treating refractory thin endometrium either without or with the Asherman syndrome. Inconsistency in the improvement of endometrial thickness is a common limitation of such therapies including tamoxifen citrate as an ovulation induction agent, acupuncture, long-term pentoxifylline and tocopherol or tocopherol only, low-dose human chorionic gonadotropin during endometrial preparation, aspirin, luteal gonadotropin-releasing hormone agonist supplementation, and extended estrogen therapy. Recently, cell therapy has been proposed as an ideal alternative for endometrium regeneration, including the employment of stem cells, platelet-rich plasma, and growth factors as therapeutic agents. The mechanisms of action of cell therapy include the cytokine induction, growth factor production, natural killer cell activity reduction, Th17 and Th1 decrease, and Treg cell and Th2 increase. Since cell therapy is personalized, dynamic, interactive, and specific and could be an effective strategy. Despite its promising nature, further research is required for improving the procedure and the safety of this strategy. These methods and their results are discussed in this article.
\end{abstract}

Keywords: Cell therapy, Endometrium, Asherman syndrome

\section{Introduction}

The route through which thin endometrium reduces the chance of pregnancy is not well-known. However, two proposed hypotheses discussing the fact that the embryo could be close to the basal layer, which is rich in reactive oxygen species and thus detrimental to the implantation and development of the embryo [1]. One or two among three women who undergo in vitro fertilization and embryo transfer are affected by a thin endometrium $(>7 \mathrm{~mm})$. One attributed cause could be the malfunctioning of endometrial stem/progenitor cells

\footnotetext{
*Correspondence: Yousefime@tbzmed.ac.ir; jahmadian@yahoo.com ${ }^{\dagger}$ Nastaran Gharibeh and Leili Aghebati-Maleki have contributed equally to this work

2 Stem Cell Research Center, Tabriz University of Medical Sciences, Tabriz, Iran

${ }^{4}$ Department of Allergy and Clinical Immunology, Pediatric Hospital, Tabriz University of Medical Sciences, Tabriz, Iran

Full list of author information is available at the end of the article
}

of the incompetent thin endometrium [2]. Satisfactory endometrium growth is an essential factor for successful implantation since low implantation rates have been reported to be associated with thin endometrium. Further, a thin endometrium is reported to be a defective cause of endometrial development. Endometrium recovery in thin endometrium patients is time-consuming although low-dose aspirin treatment or estrogen therapy has been assessed in this regard [3].

The development of the human endometrium is typically observed through transvaginal ultrasound in assisted reproductive technology cycles. A well-established marker for the receptivity of the uterus is the thickness of the endometrium [4]. The minimal cutoff endometrial thickness proposed for a successful embryo transfer is $7 \mathrm{~mm}$, even though there exists no standardized value for diagnosing a thin endometrium [5]. In addition, values above $9 \mathrm{~mm}$ could predict greater implantation rates. In fact, persistent thin endometrium original author(s) and the source, provide a link to the Creative Commons licence, and indicate if changes were made. The images or other third party material in this article are included in the article's Creative Commons licence, unless indicated otherwise in a credit line to the material. If material is not included in the article's Creative Commons licence and your intended use is not permitted by statutory regulation or exceeds the permitted use, you will need to obtain permission directly from the copyright holder. To view a copy of this licence, visit http://creativecommons.org/licenses/by/4.0/. The Creative Commons Public Domain Dedication waiver (http://creativeco mmons.org/publicdomain/zero/1.0/) applies to the data made available in this article, unless otherwise stated in a credit line to the data. 
in the reproductive cycle could be related to lower rates of implantation as well as increased rates of miscarriages [6]. Consequently, many researchers have sought to identify therapeutic modalities capable of improving endometrial receptivity and growth in endometrium-thin women [7]. Although numerous approaches (i.e., granulocyte colony-stimulating factor, sildenafil, or low-dose aspirin) have been attempted clinically, their results are inconclusive [8]. On the other hand, an important outcome of endometrial trauma is the intrauterine adhesion (IUA), resulting in the partial or full obstruction of the cervical canal or uterine cavity. Asherman syndrome (AS) defines the IUA, which is characterized by infertility, recurrent abortion, hypomenorrhea, pelvic pain, or menopause [9]. Operation procedures could generally lead to IUA through inducing artificial traumas to the uterine cavity. For instance, hysteromyomectomy, cesarean section, and curettage could injure the endometrial basal layer. Moreover, infections such as tuberculosis can cause chronic endometrial inflammation, which is in fact an inducer of adhesion [9].

The incidence rate of the AS is approximately 1.5\% [10], and the hysteroscopy adhesion lysis is regarded as the current prevalent treatment approach for this condition [11]. Nevertheless, susceptibility to anomalous placenta development and preterm delivery exists in patients following adhesion lysis due to impaired endometrial angiogenesis and metabolism [12]. It is essential to prevent adhesion in the uterine cavity post-invasive operations since two-thirds of AS-suffering women have experienced post-abortion/miscarriage curettage [13]. This could be performed through placing an intrauterine device [14]/Foley's catheter balloon/hyaluronic acid in the cavity of the uterus or employing conjugated estrogen treatment for facilitating the recovery of the endometrium [15].

Cell therapy, as one of the most effective fields of translational medicine, is an interdisciplinary field encompassing regenerative medicine, transplantation biology, biomaterials, molecular biology, tissue engineering, immunology, stem cell biology, and clinical research [14]. Cell-based therapy possesses the potential to turn into a novel therapeutic platform for treating a wide range of clinical disorders. Two major examples of cell-based therapeutics include bone marrow transplantation and blood transfusions [16]. Although recombinant genetic engineering has been effective in producing manifold therapeutics (e.g., human insulin and erythropoietin), such treatments are not capable of entirely correcting or reversing disease states [17]. This is because disease processes mostly involve changes in the multifaceted interactions of different cell components rather than a deficiency in a single protein. Under such circumstances, cell-based therapy might prove more effective through the provision of an individualized, interactive, and dynamic therapeutic approach responding to the pathophysiological condition of the patient [18]. The most recent approaches for the treatment of thin endometrium and the AS will be thoroughly reviewed in the following sections (Fig. 1).

\section{Platelet-rich plasma (PRP)}

As an available option, autologous PRP has been prominent for its safety and has been employed since the 1970s. The safety of therapy through the infusion of PRP is attributed to the fact that it is prepared from autologous blood, which itself is obtained from a peripheral vein. More importantly, preparation procedures are painless for the patient, affordable, and convenient. Since PRP releases numerous chemokines, cytokines, and growth factors stored in the alpha granules of platelets, it is capable of improving tissue regeneration [19]. Processes affected by these molecules include angiogenesis, the remodeling of the extracellular matrix, as well as the differentiation, proliferation, and recruitment of stem cells [20]. Therefore, PRP is applicable in many regenerative medicine fields such as dermatology, ophthalmology, plastic surgery, and orthopedics for promoting tissue growth and repair [21-23].

However, immunogenic and transmission reactions could be prevented since PRP is derived from autologous blood. Moreover, PRP treatment is regarded as safe according to the results of the treatment of thousands of individuals with PRP following oral-maxillary surgery [24]. More precisely, infection and other adverse effects are reported to be rare in patients. Overall, there exist several factors that could potentially determine the success of this treatment, including the selection of patients and preparation of proper cell [25].

So far, there has been limited information with regard to the potential that the application of PRP might hold in the restoration of damaged endometrium [26]. However, it has been reported that treatment with PRP led to the enhanced thickness of the endometrium (ultrasonography-approved), elevated rates of pregnancy (clinicallyapproved), and raised live-birth rates [27]. Nonetheless, it is unclear whether such improvements were directly related to PRP treatment. Further, the inadequacy of the experimental evidence could be attributed to the lack of objective validity. In an in vivo study, treatment with autologous PRP resulted in enhanced endometrium regeneration in female rats, which was confirmed by real-time polymerase chain reaction assays related to the expression of endometrial factors [28]. In another study on murine AS models without or with the infusion of human PRP, Kim et al. assessed the capability for regenerating the endometrium and the mating 


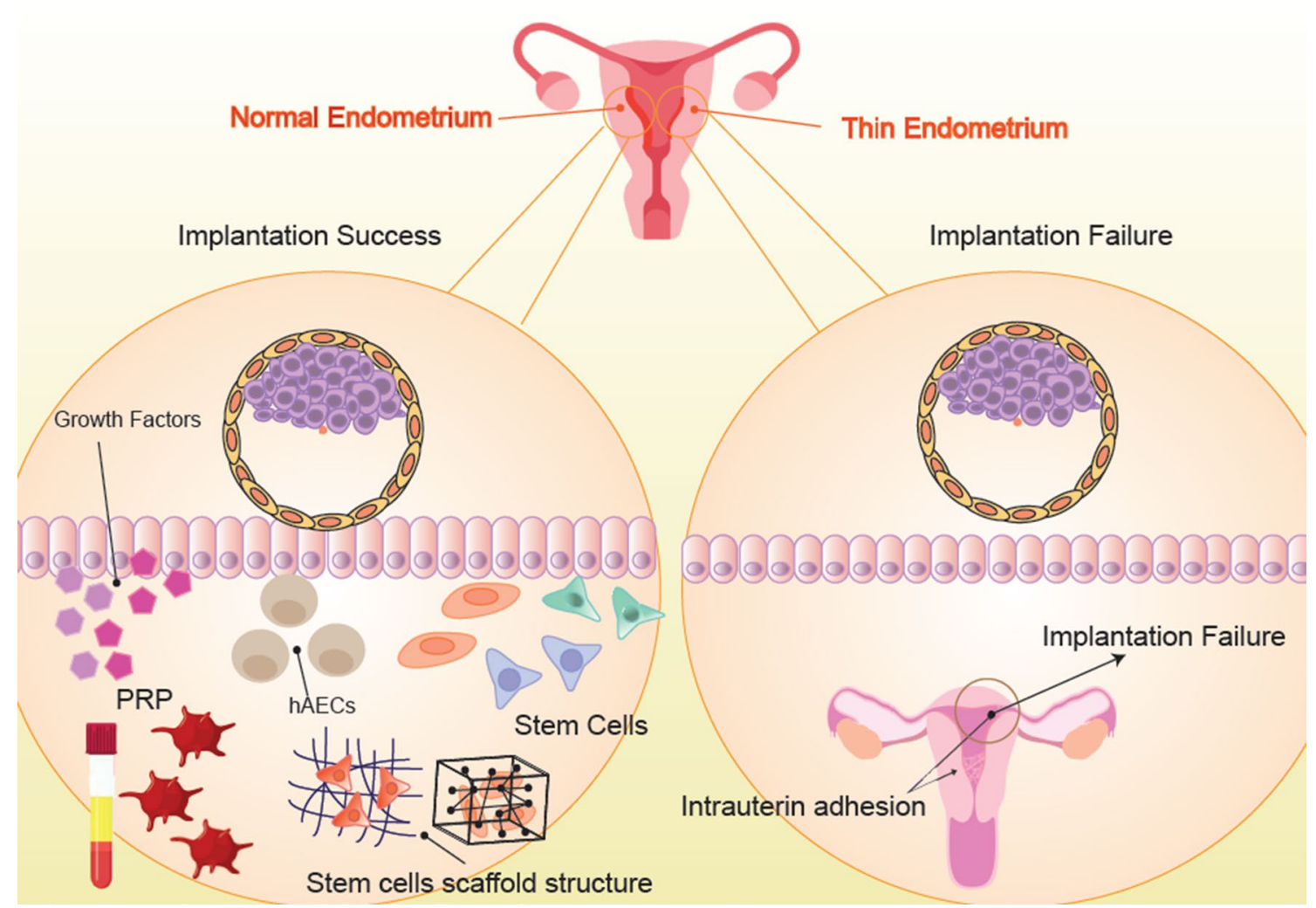

Fig. 1 On the right side of the figure, a patient with a thin endometrium is illustrated who has had implantation failure. On the left side of the figure, different types of cell therapy, which lead to the thickness of the patient's endometrium and subsequently success in pregnancy, are shown

outcomes. However, data could not distinctively imply that the donor's hormonal profile could exert effects on the therapeutic potential of his/her PRP. PRPs from separate donors showed comparable results, emphasizing that the PRP is capable of restoring damaged endometrium. Importantly, the quantification of successful pregnancy and implantation outcomes were evaluated as well. The treatment group with PRP considerably enhanced the IS number and supported the capability of carrying the pregnancy to term, resulting in an $83.3 \%$ live-birth in PRP-treated AS mice while other AS mice failed the delivery [29].

In an experiment, Wang et al. [30] investigated 20 women suffering from thin endometrium and undergoing failed embryo implantation under conventional estrogen therapy, resulting in the cancellation of the cycle and low pregnancy. Following PRP infusion, the majority of patients showed effective pregnancy and expansion of endometrium. Ultrasonography was used to evaluate the thickness of the endometrium, followed by exploring the effects of PRP on biological functions. Accordingly, PRP was capable of promoting the migration and proliferation of endometrial mesenchymal stem cells (EnM$\mathrm{SCs}$ ), subsequently differentiating into endometrial cells.
Moreover, it could be stated that PRP stimulated adhesion to Matrigel and the spreading of cells. Additional examples of PRP treatment are provided in Table 1.

\section{Growth factors}

As naturally occurring substances, growth factors (GFs) have the potential to stimulate cellular differentiation, wound healing, and cell proliferation [65] and are typically regarded as intercellular signaling molecules. For instance, hormones or cytokines are capable of binding to particular surface receptors of target cells, which frequently promote cellular maturation and differentiation differently. Epidermal growth factor (EGF) is capable of enhancing osteogenic differentiation, whereas vascular endothelial and fibroblast GFs are capable of stimulating angiogenesis (differentiation of blood vessels) [66]. As a hematopoietic growth factor, granulocyte-colonystimulating factor (G-CSF) has been demonstrated to be effective in non-hematopoietic cells such as the endometrium [67]. After the successful treatment of four individuals, it was postulated that intrauterine G-CSF may play a direct role in the promotion of endometrial growth [68]. A study on thin-endometrium patients revealed that intrauterine G-CSF administered 6-12 h before human 


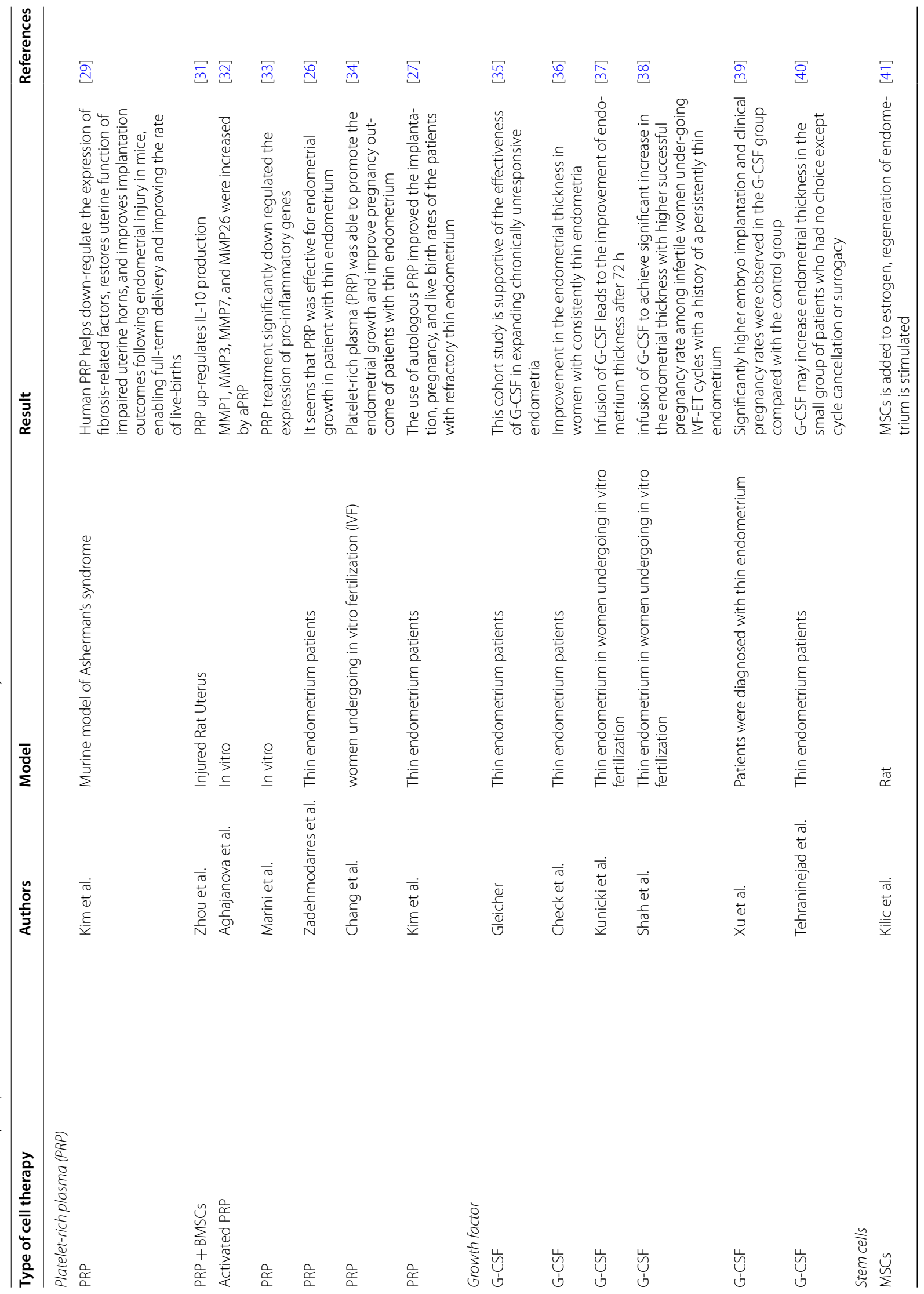




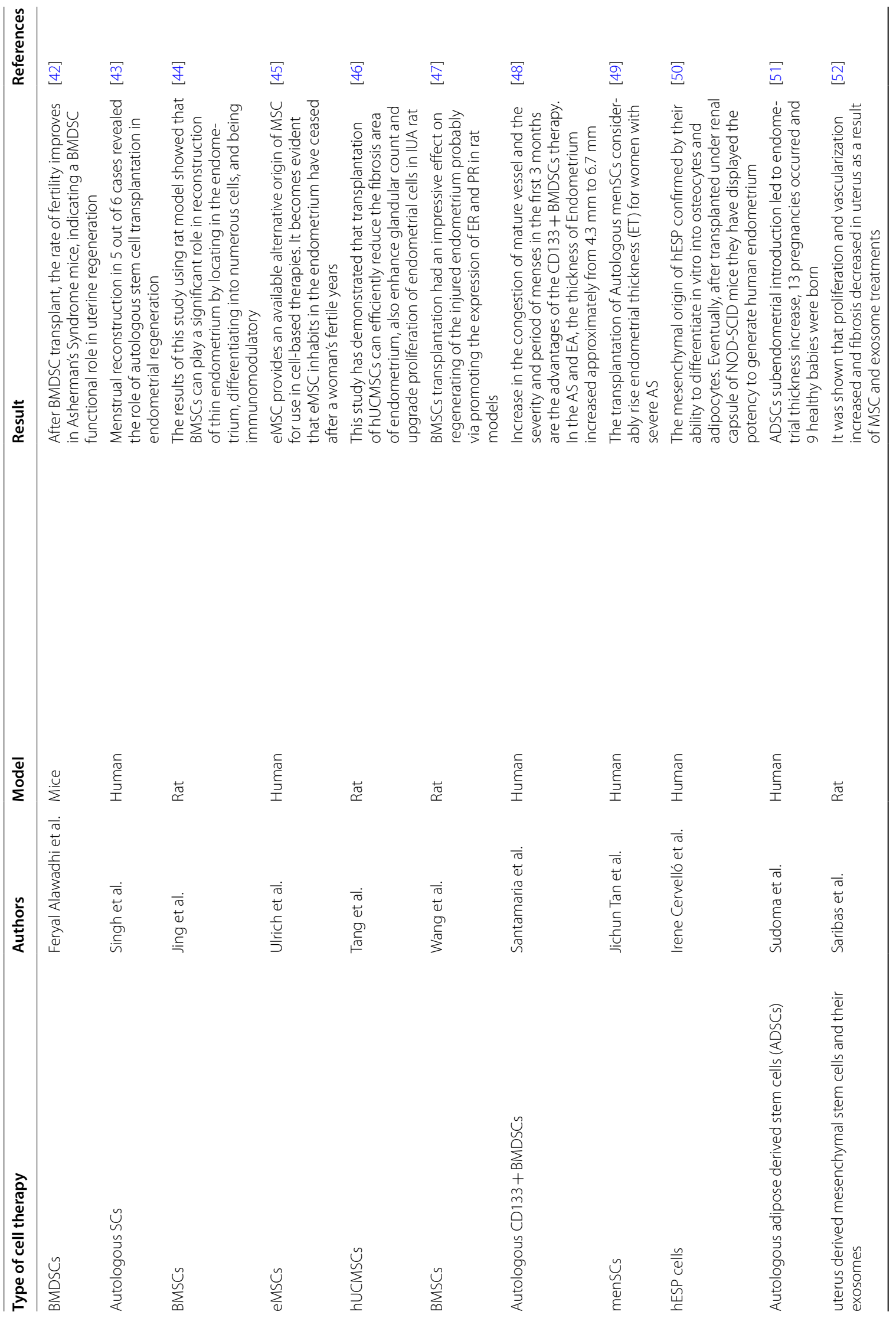




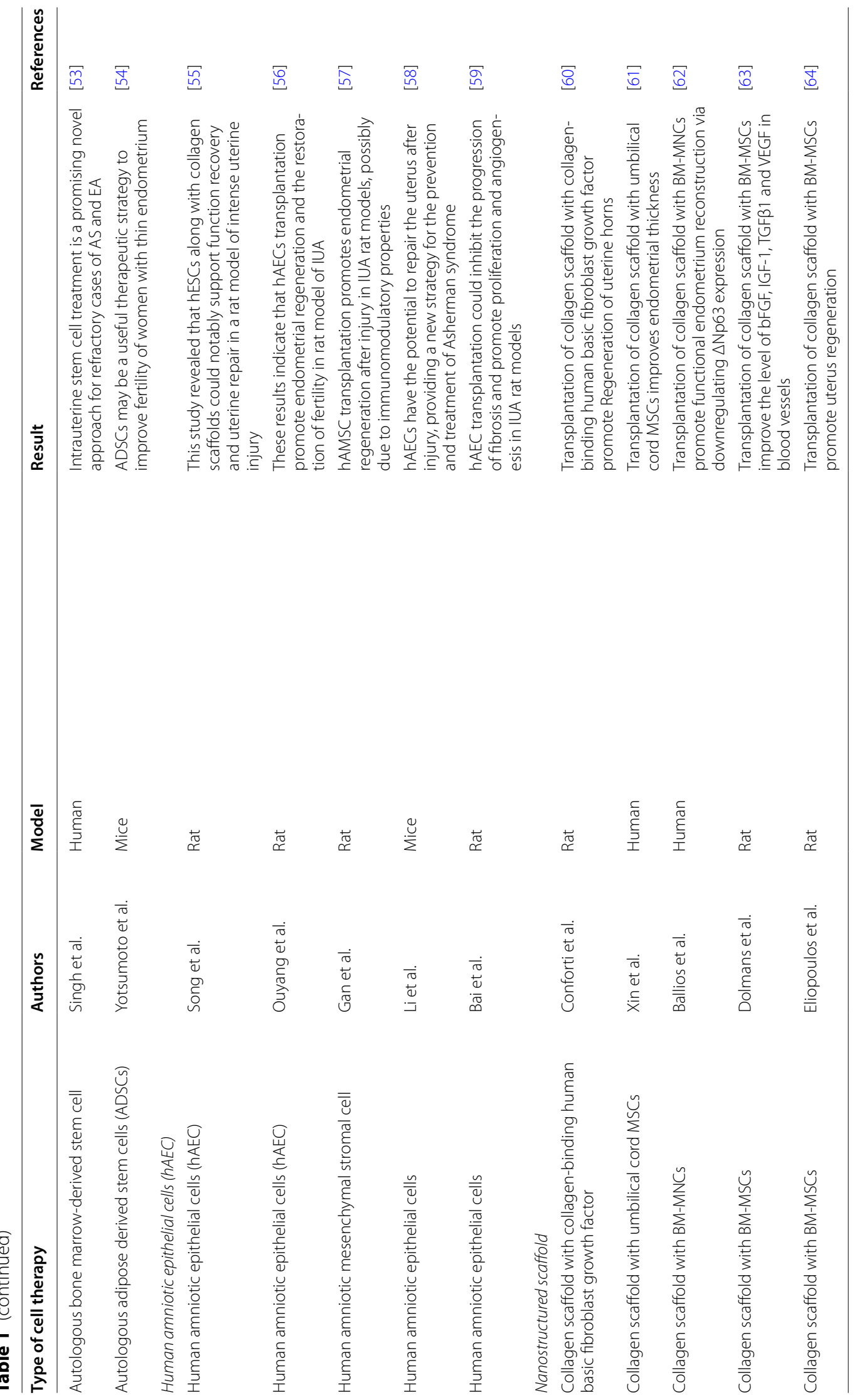


chorionic gonadotropin (HCG) trigger could significantly improve endometrial thickness, resulting in an overall pregnancy rate of $19.1 \%$ [35].

In this regard, Lucena et al. [69] successfully treated one patient while Check et al. failed to do so. Following treatment, the rate of cycle cancelation caused by thin endometrium was considerably lower $(69.39 \%$ self-controlled group versus $48.75 \%$ control group vs. $17.5 \%$ treatment group, $p<0.05$ ), with a trend towards greater pregnancy and implantation rates [70]. In another study by Kunicki et al. [37], intrauterine G-CSF $(300 \mu \mathrm{g})$ was administered to patients with a $7 \mathrm{~mm}$ EMT $6-12 \mathrm{~h}$ before HCG trigger, leading to a considerable improvement in EMT after $72 \mathrm{~h}$, with a pregnancy rate of $18.9 \%$. In a recent study, Barad et al. administered intrauterine G-CSF to FET or IVF patients regardless of the thickness of the endometrium. Furthermore, in another study, a $300 \mu \mathrm{g} / \mathrm{cm}^{3}$ dose of G-CSF was given to the study group on the day of the HCG trigger, indicating no improvements in the thickness of the endometrium [71]. Similarly, intrauterine G-CSF was administered to thin lining-possessing patients $(<8 \mathrm{~mm})$ who were resistant to treatment with vaginal sildenafil or estradiol. The treatment was also expanded to patients with repeated failure of implantation who had an EMT $>8 \mathrm{~mm}$ [38]. In a study, G-CSF $(300 \mu \mathrm{g})$ was administered to the endometrial cavity of all 231 patients 10 days post-vaginal sildenafil and oral estradiol, causing a considerable improvement in EMT and a pregnancy rate of $38.07 \%$ [72]. Further examples of GF administration are provided in Table 1.

\section{Stem cells therapy}

Stem cell therapy has been recognized as an efficient therapeutic approach among numerous types of cell therapy [73]. Stem cells are capable of dividing into pluripotent and multipotent stem cells, and several studies have enumerated both advantages and disadvantages associated with their utilization [74]. It has also been reported that it is feasible to employ stem cell therapy as a therapeutic approach for treating infertility-related diseases such as thin endometrium (Fig. 2). Numerous clinical, but challenging, trials have been performed in

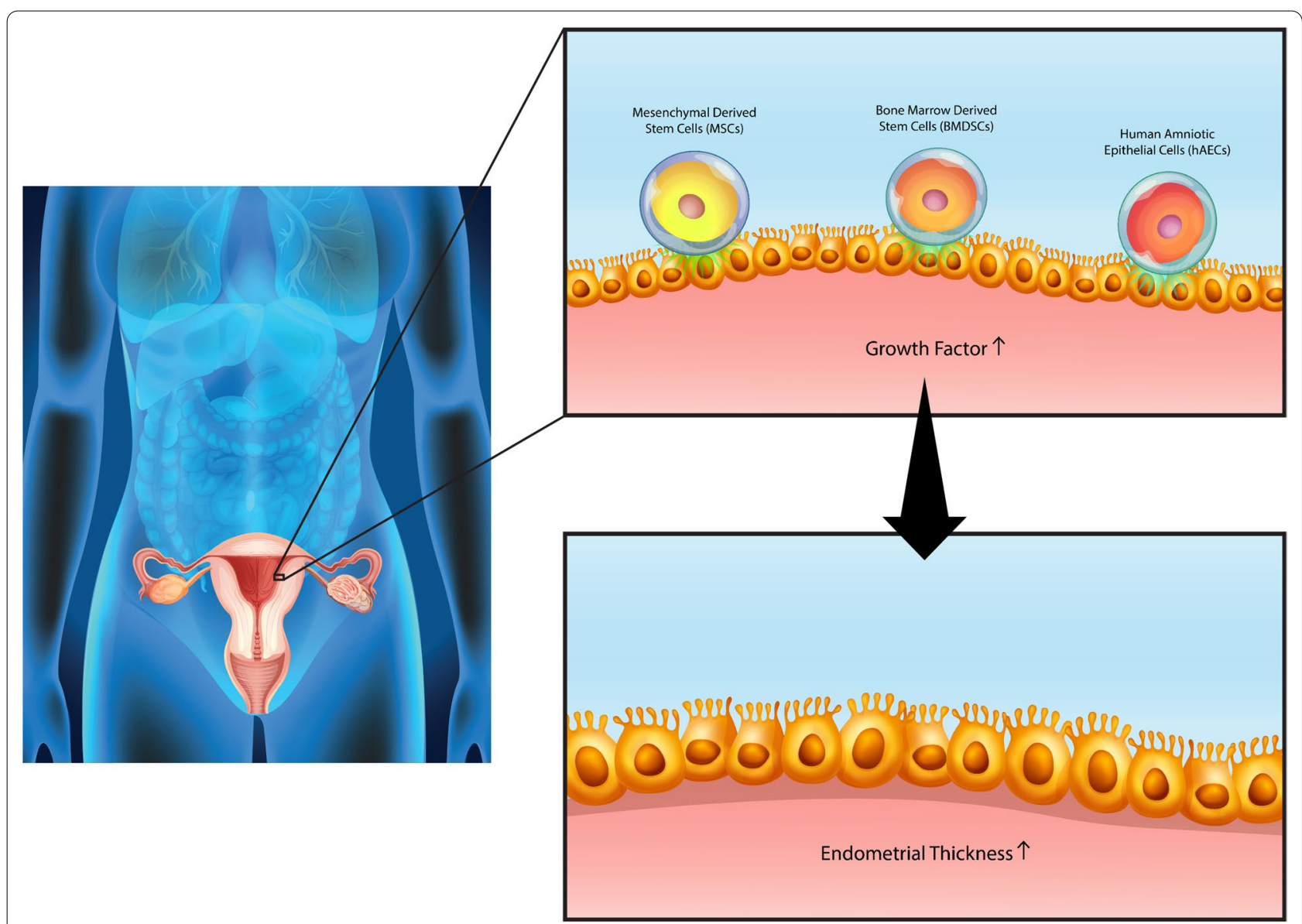

Fig. 2 Schematic of thickening of the endometrium by stem cells with the mechanism of increase in growth factors in the target area 
this regard. Overall, it seems likely that resolving issues associated with stem cell therapy could allow their application as an efficient therapeutic approach for patients suffering from infertility [75].

Similar to the majority of treatments, cell therapy could be accompanied by side effects, particularly in stem cell therapy [76]. For instance, prior to using stem cells, they should be grown for several months after they are harvested from embryos. Further, painful procedures are experienced when the aim is to obtain adult stem cells, especially from bone marrow. Moreover, stem cell treatments neither have been proven yet, nor encountered low rates of rejection [77]. Some examples of the application of stem cell therapy are summarized in Table 1.

\section{Mesenchymal derived stem cells (MSCs)}

MSCs, a type of adult stem cells, could be harvested from various tissues including bone marrow, umbilical cord, menstrual blood, endometrial tissue, adipose tissue, and the like [78]. Given the capacity of self-renewal and differentiation potentials, the emerging research has regarded MSCs as attractive candidates for cell therapy in regenerative medicine [79]. Such reactivity could reflect the tissue of origin since dissimilar sensitivity to inductive bioactive molecules in culture is exhibited by MSCs isolated from different tissues. Well-known examples include adult marrow-derived MSCs, which are frequently employed as a standard type of MSCs. Conditions which induce marrow-derived MSCs differ from those of fat-derived MSCs, which could be attributed to the presence of different microenvironments in the tissue side of the vasculature within which the pericytes reside [80]. Tissue regeneration has experienced considerable progress since the application of MSCs [81]. Nonetheless, extremely functional in vitro assays have validated the encouraging regenerative potentials of MSCs. Protective effects exerted by MSCs following allogeneic transplantation have been reported in several injured models such as damaged, neural, myocardial, hepatic, cartilage, and bone tissues [82]. It is rather recognized that the therapeutic effects of MSCs are mainly due to their immunomodulatory function, which is performed along with anti-inflammatory effects through the regulation of lymphocytes of both adaptive and innate immune systems. Further, it has been established that MSCs are capable of regulating the immune responses in numerous diseases [83]. In fact, MSCs are capable of regulating the function and proliferation of $\mathrm{T}$ cells, balancing the activity of Th2 and Th1, up-regulating Tregs functions, suppressing the functions of $\mathrm{B}$ cells, inhibiting the function and proliferation of NK cells, and preventing the activation and maturation of dendritic cells [84]. Moreover, MSCs are capable of stimulating proliferation and the secretion of cytokines in innate lymphoid cells, which are a novel family of lymphocyte-like cells playing significant roles in the innate defenses against pathogens. The regulation of the immunomodulatory functions of MSCs is performed with regard to the inflammatory conditions of their microenvironment [85]. The intensity and type of the inflammatory stimuli that are presented on MSCs determine MSC plasticity in immunomodulation. As an example, MSCs are capable of suppressing the polarization of Th17 and Th1 and promoting the polarization of Th2 in graft-versus-host disease [86]. Moreover, MSCs are capable of inhibiting Th2-dominant allergy through the inhibition of the production of IL-13 and IL-4. MSCs contribute to the fibrotic process or immunosuppressive effects under chronic and acute inflammatory conditions, respectively. Therefore, MSCs could be regarded as a feasible and flexible strategy to treat several diseases, according to their immunomodulatory characteristics [87]. However, MSCs function deteriorates with age, which might be associated with the loss of tissue homeostasis, resulting in aging-related diseases and the malfunction of organs. Zhao et al. demonstrated that injecting bone marrow mesenchymal stem cells (BMSCs) into the uterine cavities of rats resulted in a thicker endometrium as well as the upregulation of vimentin and cytokeratin as marker proteins of endometrial cells. Resultantly, directly infusing BMSCs could protect the thin endometrium rats against cell damage, in addition to promoting endometrial cell regeneration. Post-BMSC transplantation, the expression of LIF and integrin $\alpha \beta 3$ represented a considerable increase. It has been well established that LIF and integrin as the regulators of the endometrial function are the markers of the receptivity of the endometrium and possess significant parts in embryo implantation [88]. In another study [44], it was reported that the MSCs improved endometrium thickness probably via their migration and immunomodulatory properties. Table 1 provides additional examples of MSCs in the endometrium.

\section{MSC-derived extracellular vesicles (MSC-EVs)}

On the other hand, recent studies have also focused on the investigation of exosomes secreted from mesenchymal stem cells. Exosomes are active paracrine components with a high potential for repairing damaged tissue. Exosomes include many paracrine factors responsible for regeneration and angiogenesis [52]. MSC-EVs play a critical role in treating reproductive diseases such as the AS. EVs are lipid bilayer complexes that function as mediators by transferring multiple molecules to recipient cells such as proteins, microRNAs, lipids, and cytokines. Although a consensus has been reached on the mechanisms underlying MSC-EVs, several theories have been 
proposed, including promoting angiogenesis, anti-fibrosis, immunomodulation, and anti-oxidative stress levels. Moreover, numerous questions need to be fully clarified before the application of MSC-EVs in the clinic, including standardized purification and identification methods, appropriate storage and transportation systems, determined cargo for large-scale generation, and safety issues. In addition, limited yield is one of the main problems restraining the wide-spreading application of MSC-EVs. Generally, MSC-EVs have exhibited their potentials in regenerative medicine not only for their propensity ability originated from the parent cells but also for the higher biology stability and lower immunogenicity as compared to MSCs [89].

\section{Bone marrow-derived stem cells (BMDSCs)}

Being able to travel to distant organs, BMDSCs make a contribution to the regeneration and repair of tissues [90]. Since BMDSCs exist in both murine and human endometrium, it could be inferred that they have the potential for serving as a reparative cell source for the reproductive tract [91]. Regional signals of injury probably play a significant part in mobilizing BMDSCs to injured tissues. In fact, it is demonstrated that ischemia/ reperfusion injury in the uterus enhances the migration and engraftment of BMDSCs in the endometrium [92].

Alawadhi et al. reported that by the third estrous cycle after BM transplantation, female mice were bred for three months. Nine out of ten mice were placed in the BM transplant group, whereas only three mice were conceived in the non-BM transplant group (Chi-square $p=0.0225)$. On the other hand, $10 / 10$ mice were conceived in the control group, within which there was no uterine injury. The mean litter size in the BM transplant, the non-BM transplant, and control groups was 6.361.4, 5.364.0, and 7.062.0, respectively. The results indicated no significant differences between groups $(p=0.05)$ and in the time to conception between groups [42]. Further examples in this regard are presented in Table 1.

\section{Human amniotic epithelial cells (hAECs)}

As the potential stem cell source, hAECs are isolated from the amniotic membrane, which is in contact with the amniotic fluid and is the closest layer to the fetus. The immunomodulatory effect of hAECs on adaptive and innate immune cells has been reported by many studies. In addition, they are capable of differentiating into numerous cells with mesoderm and ectoderm origin, including neural cells, pancreatic cells, hepatocytes, adipocytes, cardiomyocytes, and myocytes. Further, hAECs are extremely able to suppress the proliferation of $\mathrm{B}$ cells and inhibit the migration of neutrophils and macrophages [57]. Moreover, hAECs inhibit the activation of
$\mathrm{CD} 4+\mathrm{T}$ cells and decrease the proinflammatory cytokine production of $\mathrm{CD} 4+\mathrm{T}$ cells. It has been reported that hAECs considerably enhance proliferative cell nuclear antigen (PCNA), which is responsible for accurate DNA duplication [93]. Comparing samples of the endometrium from reproductive-age women, Niklaus et al. revealed that PCNA was most abundant at the proliferative phase in both epithelial and stroma tissues. However, it reduced at the secretory phase expression in murine endometrium, suggesting the fact that hAECs may be capable of improving endometrial proliferation. Being mostly expressed throughout the proliferative phase and menstrual period, vascular endothelial growth factor (VEGF) is associated with maintaining and formulating microvessels, as well as reconstructing the endometrial tissue [94]. Chen et al. demonstrated that hysteroscopy adhesion lysis in combination with hormone replacement therapy in IUA patients significantly enhanced the expression of endometrial VEGF and MVD. Moreover, the ones with better curative effects did have greater expression of VEGF and denser microvessels as compared to patients whose responses to the treatment were poor [95]. Zhou et al. found that hAECs were capable of increasing the expression of VEGF in IUA models, demonstrating the angiogenesis potential of hAECs which may improve the recovery of endometrial injuries. Oestrogen receptor (ER), as a nuclear transcription factor, combined with estrogen is capable of promoting the proliferation and metabolism in endometrial cells [96]. In the repaired endometrium of allogeneic UCMSC-treated individuals, the expression of ER is considerably enhanced [97]. In line with this study, hAECs were reported to be able to increase the expression of ER in damaged murine endometrium. Overall, ER status may direct the regulation of endometrial injury repair [98]. Additional examples of hAEC treatment are presented in Table 1.

\section{Nanostructured scaffold}

Tissue engineering has recently attracted considerable attention. Biomaterials science is now capable of directing cellular differentiation, as opposed to its emergence, when it was only a cell carrier tool [99]. In fact, through molding, biomaterials could be utilized for synthesizing three-dimensional scaffolds capable of promoting cell differentiation and/or proliferation for regeneration [100]. Stem cell activities are significantly affected by extracellular forces, micro-geometry, matrix nano-topography, and matrix stiffness as mechanical factors. Depending on their source of derivation, biomaterials could be classified into synthetic and natural polymers. Among natural scaffolds, we can refer to keratin, chitosan, alginate, silk fibroin, collagen, and de-cellularized tissues such as de-epithelialized human amniotic membrane [101]. 
Following the transplantation of BM-MSCs-loaded collagen, it was reported that MSCs are primarily located in the basal layer of regenerative endometrium although it should be mentioned that numerous cells migrate to the wound sites [102]. Interestingly, the injured endometrium prompted BM-MSCs arrangement such that they became capable of playing key roles in remodeling a new functional endometrium. Cells in the proximity of the implantation site of BM-MSCs/collagen constructs exhibited a greater amount of vascular endothelial growth factor VEGF, transforming growth factor (TGFb1), and insulin-like growth factor (IGF-1) compared with the spontaneous regeneration group and the collagen/PBS group. Such growth factors are necessary to the regeneration of the endometrial cycle [103]. Hyaluronic acid (HA) is reported to be utilized in regenerating the endometrium in several damaged models [104]. In fact, there exists a correlation between the endometrial receptivity and the HA level for pre-implanting embryos. This result is in line with the high HA concentration in the remodeling tissues [105].

Since HA can interact with the extracellular matrix and contributes to the matrix molecules, endometrial stromal cell-loaded HA hydrogels are presumably the most suitable candidates for endometrial regeneration. It has been suggested that HA receptors exist throughout each estrous cycle, even though their contents might differ from cycle to cycle [106]. HA/fibrin hydrogels have been synthesized utilizing concentrations of $\mathrm{T}$ optimized for accelerating cross-linking and facilitating the delivery of well-conditioned cells. Since such conditioning could exert effects on the efficiency of treatment, a study examined the effect of different $\mathrm{T}$ concentrations on stiffness for constructing the most suitable platform [107]. Further examples of scaffolds in this regard are provided in Table 1.

\section{Conclusion}

As a global disease, infertility affects a great number of females and is both a social and medical issue. Given the vital role of endometrium in maternal health and reproduction, we believe that maintaining its physiological structure, eliminating its defects, and restoring it after injuries are of paramount importance. Likewise, stem cells have been the subject of many studies due to their effective functions. Therefore, we could deduce that stem cell therapy could be introduced for treating damaged tissues, cancer, and degenerative diseases as conditions with limited therapeutic options. Considering its recent advances, cell therapy has been proposed to be capable of treating numerous diseases including thin endometrium and AS. Various strategies have been introduced in stem cell therapy for boosting the survival of transplanted cells. In fact, there exist biomaterials such as nanostructure lipid carriers, hydrogels, and scaffolds, which are capable of promoting stem cell/drug delivery and thus improving the outcomes of stem cell therapy. Moreover, nano-engineered titanium implants could play vital parts in controlling stem cell/drug release. To regenerate the endometrium, we propose that the human umbilical cord mesenchymal seems to be the best clinical option as it is easily accessible, has rapid self-renewal features, is harvested abundantly through noninvasive procedures, and has low immunogenic effects. These characteristics are also common among other cell types such as PRP and hAEC. In our opinion, despite the advances in the field of cell therapy, major concerns still exist. For instance, it has been reported that endometrial stem cells are associated with the pathogenesis of many gynecological diseases (e.g., endometriosis, endometrial hyperplasia, and endometrial cancer). Other concerns as we believe include the fact that stem cells are capable of stimulating angiogenesis through the secretion of the growth factor. Overall, we could state that these novel therapies might actually enjoy numerous advantages over the traditional therapies in this regard. However, we understand that there still exists room for improvement, and much research is required to maximize the potentials of emerging novel therapies. Finally, with the advent of new cell therapies and the potential for infertility treatment, we are hopeful that many of the problems in this area will be resolved soon, especially in developing countries.

\section{Abbreviations \\ ART: Assisted reproductive technology; AS: Asherman syndrome; BMSCs: Bone marrow mesenchymal stem cells; EGF: Epidermal growth factor; EMT: Extra matrix transmission; EnMSCs: Endometrium mesenchymal stem cells; FET: Frozen-thawed embryo transfer; GCSF: Granulocyte colony-stimulating factor; GFS: Growth factors; GnRH: Gonadotropin-releasing hormone; GVHD: Graft- versus-host disease; hAECs: Human amniotic epithelial cells; HCG: Human chorionic gonadotropin; HRT: Hormone replacement therapy; IGF: Insulin-like growth factor; IUA: Intrauterine adhesion; MSCs: Mesenchymal derived stem cells; NK: Natural killer cell; PBMC: Peripheral blood mononuclear cell; PRP: Platelet-rich plasma; TGF: Transforming growth factor; Th: Tcell helper; TNF-a: Tumor necrosis factor-a; VEGF: Vascular endothelial growth factor.}

\section{Acknowledgements}

This work was supported by the Stem Cell Research Center (SCRC), Tabriz University of Medical Sciences, Tabriz, Iran.

\section{Authors' contributions}

NG and JM have made contributions to the writing of the manuscript, the design of the figures. RP and LAM participated in literature survey. LAM and MY contributed to the study design and revised of the manuscript. JAH contributed to the revised of the manuscript. All authors have approved the submitted version of the article and have agreed to be personally accountable for the author's own contributions and to ensure that questions related to the accuracy or integrity of any part of the work. All authors read and approved the final manuscript.

Funding

Not applicable. 


\section{Availability of data and materials}

Data sharing is not applicable to this article as no new data were created or analyzed in this study.

\section{Declarations}

\section{Ethics approval and consent to participate}

Not applicable.

\section{Consent for publication}

Not applicable.

\section{Competing interests}

The authors declare that they have no competing interests.

\section{Author details}

'Student's Research Committee, Tabriz University of Medical Sciences, Tabriz, Iran. ${ }^{2}$ Stem Cell Research Center, Tabriz University of Medical Sciences, Tabriz, Iran. ${ }^{3}$ Immunology Research Center, Tabriz University of Medical Sciences, Tabriz, Iran. ${ }^{4}$ Department of Allergy and Clinical Immunology, Pediatric Hospital, Tabriz University of Medical Sciences, Tabriz, Iran. ${ }^{5}$ Department of Immunology, School of Medicine, Tabriz University of Medical Sciences, Tabriz, Iran.

Received: 8 June 2021 Accepted: 24 December 2021

Published online: 28 January 2022

\section{References}

1. Armstrong GM, et al. Endometrial apoptosis and neutrophil infiltration during menstruation exhibits spatial and temporal dynamics that are recapitulated in a mouse model. Sci Rep. 2017;7(1):1-14.

2. Liu K, et al. The impact of a thin endometrial lining on fresh and frozenthaw IVF outcomes: an analysis of over 40,000 embryo transfers. Hum Reprod. 2018;33(10):1883-8.

3. Maekawa $\mathrm{R}$, et al. Thin endometrium transcriptome analysis reveals a potential mechanism of implantation failure. Reprod Med Biol. 2017;16(2):206-27.

4. Adamson GD, et al. International Committee for Monitoring Assisted Reproductive Technology: world report on assisted reproductive technology, 2011. Fertil Steril. 2018;110(6):1067-80

5. Hou X, et al. Endometrial regeneration in Asherman's syndrome: clinical and translational evidence of stem cell therapies. Curr Stem Cell Res Ther. 2019;14(6):454-9.

6. Bashiri A, Halper Kl, Orvieto R. Recurrent implantation failure-update overview on etiology, diagnosis, treatment and future directions. Reprod Biol Endocrinol. 2018;16(1):1-18.

7. Da Broi M, et al. Is the profile of transcripts altered in the eutopic endometrium of infertile women with endometriosis during the implantation window? Hum Reprod. 2019:34(12):2381-90.

8. Ranisavljevic N, et al. Embryo transfer strategy and therapeutic options in infertile patients with thin endometrium: a systematic review. J Assist Reprod Genet. 2019;36:1-15.

9. Santamaria X, Isaacson K, Simón C. Asherman's syndrome: it may not be all our fault. Hum Reprod. 2018;33(8):1374-80.

10. Dreisler E, Kjer JJ. Asherman's syndrome: current perspectives on diagnosis and management. Int J Women's Health. 2019;11:191.

11. Salazar CA, Isaacson K, Morris S. A comprehensive review of Asherman's syndrome: causes, symptoms and treatment options. Curr Opin Obstet Gynecol. 2017;29(4):249-56

12. Lekovich J, et al. Placental malperfusion as a possible mechanism of preterm birth in patients with Müllerian anomalies. J Perinat Med. 2017;45(1):45-9.

13. Khan Z, Goldberg JM. Hysteroscopic management of Asherman's syndrome. J Minim Invasive Gynecol. 2018;25(2):218-28.

14. Pourakbari $\mathrm{R}$, et al. The potential of exosomes in the therapy of the cartilage and bone complications; emphasis on osteoarthritis. Life Sci. 2019:236:116861.
15. Johary J, et al. Efficacy of estrogen therapy in patients with intrauterine adhesions: systematic review. J Minim Invasive Gynecol. 2014:21(1):44-54

16. Ye Z, Mahato RI. Role of nanomedicines in cell-based therapeutics. 2008

17. Gage FH. Cell therapy. Nature. 1998;392(6679 Suppl):18-24

18. Pourakbari $R$, et al. Cell therapy in female infertility-related diseases: emphasis on recurrent miscarriage and repeated implantation failure. Life Sci. 2020:258:118181.

19. Etulain J, et al. An optimised protocol for platelet-rich plasma preparation to improve its angiogenic and regenerative properties. Sci Rep. 2018;8(1):1-15

20. Farimani $M$, et al. Successful pregnancy and live birth after intrauterine administration of autologous platelet-rich plasma in a woman with recurrent implantation failure: a case report. Int J Reprod BioMed. 2017:15(12):803.

21. Tian J, et al. The effects of aging, diabetes mellitus, and antiplatelet drugs on growth factors and anti-aging proteins in platelet-rich plasma. Platelets. 2019;30(6):773-92.

22. Meftahpour $\mathrm{V}$, et al. Platelet lysate: a promising candidate in regenerative medicine. Regen Med. 2021;16(1):71-85.

23. Fotouhi $\mathrm{A}$, et al. Platelet rich plasma, stromal vascular fraction and autologous conditioned serum in treatment of knee osteoarthritis. Biomed Pharmacother. 2018;104:652-60.

24. Franchini $M$, et al. The use of platelet-rich plasma in oral surgery: a systematic review and meta-analysis. Blood Transfus. 2019;17(5):357

25. Marx RE. Platelet-rich plasma: evidence to support its use. J Oral Maxillofac Surg. 2004;62(4):489-96

26. Zadehmodarres $\mathrm{S}$, et al. Treatment of thin endometrium with autologous platelet-rich plasma: a pilot study. JBRA Assist Reprod. 2017;21(1):54.

27. Kim H, et al. Effect of autologous platelet-rich plasma treatment on refractory thin endometrium during the frozen embryo transfer cycle: a pilot study. Front Endocrinol. 2019;10:61.

28. Jang $\mathrm{H}-\mathrm{Y}$, et al. Effects of autologous platelet-rich plasma on regeneration of damaged endometrium in female rats. Yonsei Med J. 2017;58(6):1195-203.

29. Kim JH, et al. Intrauterine infusion of human platelet-rich plasma improves endometrial regeneration and pregnancy outcomes in a murine model of Asherman's syndrome. Front Physiol. 2020;11:105.

30. Wang $X$, et al. Investigation of platelet-rich plasma in increasing proliferation and migration of endometrial mesenchymal stem cells and improving pregnancy outcome of patients with thin endometrium. J Cell Biochem. 2019;120(5):7403-11.

31. Zhou Y, et al. Platelet-rich plasma therapy enhances the beneficial effect of bone marrow stem cell transplant on endometrial regeneration. Front Cell Dev Biol. 2020:8:52-52.

32. Aghajanova $L$, et al. In vitro evidence that platelet-rich plasma stimulates cellular processes involved in endometrial regeneration. J Assist Reprod Genet. 2018;35(5):757-70.

33. Marini MG, et al. Effects of platelet-rich plasma in a model of bovine endometrial inflammation in vitro. Reprod Biol Endocrinol. 2016:14(1):1-17.

34. Chang Y, et al. Autologous platelet-rich plasma promotes endometrial growth and improves pregnancy outcome during in vitro fertilization. Int J Clin Exp Med. 2015;8(1):1286.

35. Gleicher $\mathrm{N}$, et al. A pilot cohort study of granulocyte colony-stimulating factor in the treatment of unresponsive thin endometrium resistant to standard therapies. Hum Reprod. 2013;28(1):172-7.

36. Check JH, Cohen $\mathrm{R}$, Choe J. Failure to improve a thin endometrium in the late proliferative phase with uterine infusion of granulocytecolony stimulating factor. Clin Exp Obstet Gynecol. 2014:41(4):473-5.

37. Kunicki M, et al. Evaluation of granulocyte colony-stimulating factor effects on treatment-resistant thin endometrium in women undergoing in vitro fertilization. BioMed Res Int. 2014;2014:913235.

38. Shah J, Gangadharan A, Shah V. Effect of intrauterine instillation of granulocyte colony-stimulating factor on endometrial thickness and clinical pregnancy rate in women undergoing in vitro fertilization cycles: an observational cohort study. Int J Infertil Fetal Med. 2014;5(3):100-6. 
39. Xu B, et al. Two protocols to treat thin endometrium with granulocyte colony-stimulating factor during frozen embryo transfer cycles. Reprod Biomed Online. 2015;30(4):349-58.

40. Tehraninejad E, et al. G-CSF intrauterine for thin endometrium, and pregnancy outcome. J Family Reprod Health. 2015;9(3):107.

41. Kilic $S$, et al. Effect of stem cell application on Asherman syndrome, an experimental rat model. J Assist Reprod Genet. 2014;31(8):975-82.

42. Alawadhi F, et al. Bone marrow-derived stem cell (BMDSC) transplantation improves fertility in a murine model of Asherman's syndrome. PLoS ONE. 2014;9(5):e96662.

43. Singh $\mathrm{N}$, et al. Autologous stem cell transplantation in refractory Asherman's syndrome: a novel cell based therapy. J Hum Reprod Sci. 2014;7(2):93.

44. Jing Z, et al. Rat bone marrow mesenchymal stem cells improve regeneration of thin endometrium in rat. Fertil Steril. 2014;101(2):587-94.

45. Ulrich $D$, et al. Mesenchymal stem/stromal cells in post-menopausal endometrium. Hum Reprod. 2014;29(9):1895-905.

46. Tang Y-Q, et al. Effects of human umbilical cord mesenchymal stem cells on intrauterine adhesions in a rat model. Int J Clin Exp Pathol. 2016:9(11):12119-29.

47. Wang J, et al. Application of bone marrow-derived mesenchymal stem cells in the treatment of intrauterine adhesions in rats. Cell Physiol Biochem. 2016;39(4):1553-60.

48. Santamaria X, et al. Autologous cell therapy with CD133+ bone marrow-derived stem cells for refractory Asherman's syndrome and endometrial atrophy: a pilot cohort study. Hum Reprod. 2016;31(5):1087-96.

49. Tan J, et al. Autologous menstrual blood-derived stromal cells transplantation for severe Asherman's syndrome. Hum Reprod. 2016;31(12):2723-9.

50. Cervello l, et al. Reconstruction of endometrium from human endometrial side population cell lines. PLoS ONE. 2011;6(6):e21221.

51. Sudoma I, et al. Application of autologous adipose-derived stem cells for thin endometrium treatment in patients with failed ART programs. J Stem Cell Ther Transpl. 2019;3:001-8.

52. Saribas GS, et al. Effects of uterus derived mesenchymal stem cells and their exosomes on Asherman's syndrome. Acta Histochem. 2020;122(1):151465.

53. Singh N, et al. Autologous bone marrow-derived stem cell therapy for Asherman's syndrome and endometrial atrophy: a 5-year follow-up study. J Hum Reprod Sci. 2020;13(1):31.

54. Yotsumoto F, et al. Adipose tissue-derived regenerative cells improve implantation of fertilized eggs in thin endometrium. Regen Med. 2020;15(7):1891-904.

55. Song T, et al. Regeneration of uterine horns in rats using collagen scaffolds loaded with human embryonic stem cell-derived endometriumlike cells. Tissue Eng Part A. 2015;21(1-2):353-61.

56. Ouyang $X$, et al. Transplantation of human amnion epithelial cells improves endometrial regeneration in rat model of intrauterine adhesions. Stem Cells Dev. 2020;29(20):1346-62.

57. Gan L, et al. Human amniotic mesenchymal stromal cell transplantation improves endometrial regeneration in rodent models of intrauterine adhesions. Cytotherapy. 2017;19(5):603-16.

58. Li B, et al. Human amniotic epithelial cells improve fertility in an intrauterine adhesion mouse model. Stem Cell Res Ther. 2019;10(1):1-14.

59. Bai $X$, et al. Therapeutic effect of human amniotic epithelial cells in rat models of intrauterine adhesions. Cell Transpl. 2020;29:0963689720908495.

60. Conforti A, et al. The management of Asherman syndrome: a review of literature. Reprod Biol Endocrinol. 2013;11(1):1-11.

61. Xin L, et al. A collagen scaffold loaded with human umbilical cord derived mesenchymal stem cells facilitates endometrial regeneration and restores fertility. Acta Biomater. 2019;92:160-71.

62. Ballios BG, et al. A hydrogel-based stem cell delivery system to treat retinal degenerative diseases. Biomaterials. 2010;31(9):2555-64.

63. Dolmans MM, et al. Reimplantation of cryopreserved ovarian tissue from patients with acute lymphoblastic leukemia is potentially unsafe. Blood. 2010;116(16):2908-14.

64. Eliopoulos $\mathrm{N}$, et al. Human marrow-derived mesenchymal stromal cells decrease cisplatin renotoxicity in vitro and in vivo and enhance survival of mice post-intraperitoneal injection. Am J Physiol Renal Physiol. 2010;299(6):F1288-98.
65. Chen F-M, Zhang M, Wu Z-F. Toward delivery of multiple growth factors in tissue engineering. Biomaterials. 2010;31(24):6279-308.

66. Zamani $M$, et al. Humanized culture medium for clinical-grade generation of erythroid cells from umbilical cord blood CD34+ Cells. Adv Pharm Bull. 2021:11:335.

67. Jensen $J R$, et al. A potential role for colony-stimulating factor 1 in the genesis of the early endometriotic lesion. Fertil Steril. 2010;93(1):251-6.

68. Gleicher N, Vidali A, Barad DH. Successful treatment of unresponsive thin endometrium. Fertil Steril. 2011;95(6):2123.e13-2123.e17.

69. Lucena E, Moreno-Ortiz H. Granulocyte colony-stimulating factor (G-CSF): a mediator in endometrial receptivity for a patient with polycystic ovary (PCO) undergoing in vitro maturation (IVM). Case Rep. 2013;2013:bcr2012008115.

70. Li Y, et al. Granulocyte colony-stimulating factor administration for infertile women with thin endometrium in frozen embryo transfer program. Reprod Sci. 2014;21(3):381-5.

71. Barad $\mathrm{DH}$, et al. A randomized clinical trial of endometrial perfusion with granulocyte colony-stimulating factor in in vitro fertilization cycles: impact on endometrial thickness and clinical pregnancy rates. Fertil Steril. 2014;101(3):710-5.

72. Eftekhar M, Sayadi M, Arabjahvani F. Transvaginal perfusion of G-CSF for infertile women with thin endometrium in frozen ET program: a nonrandomized clinical trial. Iran J Reprod Med. 2014;12(10):661.

73. Aghebati-Maleki $\mathrm{L}$, et al. Prospect of mesenchymal stem cells in therapy of osteoporosis: a review. J Cell Physiol. 2019;234(6):8570-8.

74. Li L, Xie T. Stem cell niche: structure and function. Annu Rev Cell Dev Biol. 2005;21:605-31.

75. Segers VF, Lee RT. Stem-cell therapy for cardiac disease. Nature. 2008:451(7181):937-42.

76. Jahanbani Y, et al. Scaffold-based tissue engineering approaches in treating infertility. Life Sci. 2020;240:117066.

77. Zakrzewski W, et al. Stem cells: past, present, and future. Stem Cell Res Ther. 2019;10(1):1-22.

78. Shareghi-oskoue O, Aghebati-Maleki L, Yousefi M. Transplantation of human umbilical cord mesenchymal stem cells to treat premature ovarian failure. Stem Cell Res Ther. 2021;12(1):1-3.

79. Nouri N, Aghebati-Maleki L, Yousefi M. Adipose-derived mesenchymal stem cells: a promising tool in the treatment of pre mature ovarian failure. J Reprod Immunol. 2021;147:103363.

80. Caplan Al. All MSCs are pericytes? Cell Stem Cell. 2008;3(3):229-30.

81. Wang S, Qu X, Zhao RC. Clinical applications of mesenchymal stem cells. J Hematol Oncol. 2012:5(1):19.

82. Atala A, Kasper FK, Mikos AG. Engineering complex tissues. Sci Transl Med. 2012;4(160):160rv12-160rv12.

83. Schwartz RE, et al. Multipotent adult progenitor cells from bone marrow differentiate into functional hepatocyte-like cells. J Clin Investig. 2002;109(10):1291-302.

84. Dai W, et al. Allogeneic mesenchymal stem cell transplantation in postinfarcted rat myocardium. Circulation. 2005;112(2):214-23.

85. Bae JS, et al. Bone marrow-derived mesenchymal stem cells promote neuronal networks with functional synaptic transmission after transplantation into mice with neurodegeneration. Stem cells. 2007:25(5):1307-16.

86. Gao F, et al. Mesenchymal stem cells and immunomodulation: current status and future prospects. Cell Death Dis. 2016;7(1):e2062-e2062.

87. Spaggiari GM, et al. Mesenchymal stem cells inhibit natural killer-cell proliferation, cytotoxicity, and cytokine production: role of indoleamine 2, 3-dioxygenase and prostaglandin E2. Blood J Am Soc Hematol. 2008;111(3):1327-33.

88. Zhao J, et al. Uterine infusion with bone marrow mesenchymal stem cells improves endometrium thickness in a rat model of thin endometrium. Reprod Sci. 2015;22(2):181-8.

89. Liao Z, et al. Therapeutic role of mesenchymal stem cell-derived extracellular vesicles in female reproductive diseases. Front Endocrinol. 2021;12(711):665645.

90. Taylor HS. Endometrial cells derived from donor stem cells in bone marrow transplant recipients. JAMA. 2004;292(1):81-5.

91. Du H, Taylor HS. Contribution of bone marrow-derived stem cells to endometrium and endometriosis. Stem Cells. 2007;25(8):2082-6.

92. Du H, Naqvi H, Taylor HS. Ischemia/reperfusion injury promotes and granulocyte-colony stimulating factor inhibits migration of 
bone marrow-derived stem cells to endometrium. Stem Cells Dev 2012;21(18):3324-31.

93. Moldovan GL, Pfander B, Jentsch S. PCNA, the maestro of the replication fork. Cell. 2007;129(4):665-79.

94. Punyadeera $C$, et al. Expression and regulation of vascular endothelial growth factor ligands and receptors during menstruation and post-menstrual repair of human endometrium. Mol Hum Reprod. 2006;12(6):367-75.

95. Chen Y, Chang Y, Yao S. Role of angiogenesis in endometrial repair of patients with severe intrauterine adhesion. Int J Clin Exp Pathol. 2013;6(7):1343-50.

96. Zhou Q, et al. Abnormal expression of fibrosis markers, estrogen receptor $\mathrm{a}$ and stromal derived factor-1/chemokine (C-X-C motif) receptor-4 axis in intrauterine adhesions. Int J Mol Med. 2018:42(1):81-90.

97. Cao Y, et al. Allogeneic cell therapy using umbilical cord MSCs on collagen scaffolds for patients with recurrent uterine adhesion: a phase I clinical trial. Stem Cell Res Ther. 2018;9(1):192.

98. Li B, et al. Human amniotic epithelial cells improve fertility in an intrauterine adhesion mouse model. Stem Cell Res Ther. 2019;10(1):257.

99. Aghlmandi A, et al. Microfluidics as efficient technology for the isolation and characterization of stem cells. EXCLI J. 2021;20:426-43.

100. Hayashi Y, et al. Biomaterials: chitosan and collagen for regenerative medicine. Biomed Res Int. 2014;2014:690485.

101. Krishna L, et al. Nanostructured scaffold as a determinant of stem cell fate. Stem Cell Res Ther. 2016;7(1):188.

102. Nikshad A, et al. Advances of microfluidic technology in reproductive biology. Life Sci. 2021;265:118767.

103. Ding $L$, et al. Transplantation of bone marrow mesenchymal stem cells on collagen scaffolds for the functional regeneration of injured rat uterus. Biomaterials. 2014;35(18):4888-900

104. Tamadon A, et al. Efficient biomaterials for tissue engineering of female reproductive organs. Tissue Eng Regen Med. 2016;13(5):447-54.

105. Afify AM, Craig S, Paulino AF. Temporal variation in the distribution of hyaluronic acid, CD44s, and CD44v6 in the human endometrium across the menstrual cycle. Appl Immunohistochem Mol Morphol. 2006;14(3):328-33.

106. Gomes J, et al. Sodium hyaluronate (hyaluronic acid) promotes migration of human corneal epithelial cells in vitro. Br J Ophthalmol. 2004;88(6):821-5

107. Vardy MD, et al. The effects of hormone replacement on the biomechanical properties of the uterosacral and round ligaments in the monkey model. Am J Obstet Gynecol. 2005;192(5):1741-51.

\section{Publisher's Note}

Springer Nature remains neutral with regard to jurisdictional claims in published maps and institutional affiliations.

Ready to submit your research? Choose BMC and benefit from:

- fast, convenient online submission

- thorough peer review by experienced researchers in your field

- rapid publication on acceptance

- support for research data, including large and complex data types

- gold Open Access which fosters wider collaboration and increased citations

- maximum visibility for your research: over $100 \mathrm{M}$ website views per year

At $\mathrm{BMC}$, research is always in progress.

Learn more biomedcentral.com/submissions 